\title{
Distributed average consensus: Beyond the realm of linearity
}

\author{
Usman A. Khan, Soummya Kar, and José M. F. Moura \\ Department of Electrical and Computer Engineering \\ Carnegie Mellon University \\ 5000 Forbes Ave, Pittsburgh, PA 15213 \\ \{ukhan,moura\}@ece.cmu.edu, soummyak@andrew.cmu.edu \\ Ph: (412)268-7103 Fax: (412)268-3890
}

\begin{abstract}
In this paper, we present a distributed averageconsensus algorithm with non-linear updates. In particular, we use a weighted combination of the sine of the state differences among the nodes as a consensus update instead of the conventional linear update which just includes a weighted combination of the state differences. The non-linear update comes from the theory of non-linear iterative algorithms that we present elsewhere. We show the non-linear average-consensus converges to the initial average under appropriate conditions on the combining weights. By simulations, we show that the convergence rate of our algorithm outperform the conventional linear case.
\end{abstract}

\section{INTRODUCTION}

Recently, there has been a significant interest in linear distributed average-consensus (LDAC) problem [1]. In this problem, we are interested in computing the average of several scalar quantities distributed over a sensor network. These scalar quantities become the initial conditions of the LDAC algorithm. In the LDAC algorithm, each sensor updates its state as a linear combination of the neighboring states. Under appropriate conditions [1], [2], the state at each sensor converges to the average of the initial conditions. So far, the focus in this research has been on linear updates, where the convergence rate only depends on the network connectivity (second largest eigenvalue of graph Laplacian).

In this paper, we introduce a distributed average-consensus algorithm with non-linear updates. The state update in the non-linear distributed average-consensus (NLDAC) consists of the sensor's previous state added to a linear combination of the sine of the state differences among the neighboring nodes. Due to the non-linearity introduced by the sine function, the convergence rate now depends on the actual states of the nodes. As will be shown in the paper, this fact makes the convergence rate of NLDAC faster, by appropriate tuning the combining weights.

The NLDAC using the sinusoids stems from our work on the theory of distributed non-linear iterative algorithms that we present elsewhere. In fact, non-linear functions other than sinusoids can also be employed, if the chosen nonlinear function has certain properties that we elaborate in the

This work was partially supported by NSF under grants \# ECS-0225449 and \# CNS-0428404, and by ONR under grant \# MURI-N000140710747. paper. The sine function can be used as a combining function in the distributed iterative algorithms since it is Lipschitz continuous with Lipschitz constant strictly less than unity if the frequency and the domain of the sine function is chosen appropriately.

In this context, we note that our work can be tied to results on networks of coupled oscillators (see, for example, [3], [4], [5]). The aforementioned works concern qualitative properties of such networks. In contrast, we propose schemes for a design of algorithms with desirable properties (in our case, average-consensus) and our methodology is different. The framework, presented here, goes beyond averageconsensus and is likely to find applications in other areas of distributed signal processing, like distributed phase-locked loops [6], large-scale power networks [7], where such form of dynamics arise naturally.

For simplicity of the exposition, we assume that each sensor, $l$, possesses a scalar quantity, $y_{l}$, such that $y_{l} \in$ $[-\pi / 4+\epsilon, \pi / 4-\epsilon] \forall l$. This is needed because we would like to operate in the domain of the sine function where the $\cos \left(y_{l}-y_{j}\right)$ (as will be shown, the cosine determines the convergence rate as it is the derivative of the sine) does not take the value 0 , for any $l$ and $j$. It is noteworthy that this assumption does not put any restriction on the algorithm when $y_{l}$ 's are arbitrary as long as they are bounded, i.e., $\left|y_{l}\right|<M, \forall l$. In such case, we can always choose an appropriate frequency, $\zeta$, such that $\cos \left(\zeta\left(y_{l}-y_{j}\right)\right)$ does not vanish and the resulting convergence rate involves an additional factor of $\zeta$.

We now describe the rest of the paper. In Section II, we recapitulate some relevant concepts from graph theory. Section III discusses the problem formulation and introduces the non-linear distributed average-consensus algorithm. We analyze the algorithm in Section IV and derive the conditions for convergence. In Section V, we present simulations and finally, Section VI concludes the paper.

\section{Preliminaries}

Consider a network of $N$ nodes where the $N$ nodes are inter-connected through an undirected communication graph, $\mathcal{G}=(V, \mathbf{A})$, where $V=\{1, \ldots, N\}$ is the set of vertices 
and $\mathbf{A}=\left\{a_{l j}\right\}$ is the adjacency matrix of the communication graph, $\mathcal{G}$. Since the graph is undirected, the adjacency matrix, $\mathbf{A}$, is symmetric. We define $\mathcal{K}(l)$ as the neighbors of node $l$, i.e.,

$$
\mathcal{K}(l) \triangleq\left\{j \mid a_{l j}=1\right\} .
$$

Similarly, we define

$$
\mathcal{D}_{l} \triangleq\{l\} \cup \mathcal{K}(l) .
$$

Let $K$ be the total number of edges in $\mathcal{G}$. Let $\mathbf{C}=$ $\left\{c_{l k}\right\}_{l=1, \ldots, N}^{k=1, \ldots, K}$ be the $N \times K$ incidence matrix of $\mathcal{G}$ where its $k$ th column represents the $k$ th edge, $(i, j) \in \mathcal{G}$, such that $c_{i k}=1$ and $c_{j k}=-1$. The Laplacian, $\mathbf{L}$, of $\mathcal{G}$ is then defined as

$$
\mathbf{L}=\mathbf{C C}^{T} .
$$

If $w_{k}$ is a weight associated to the $k$ th edge in $\mathcal{G}$, then the weighted Laplacian matrix is defined as

$$
\mathbf{L}_{w}=\mathbf{C W C}^{T},
$$

where $\mathbf{W}$ is a $K \times K$ diagonal matrix such that the $k$ th element on its diagonal (that represents the $k$ th edge in $\mathcal{G}$ ) is $w_{k}$.

Note that the Laplacian, $\mathbf{L}$, is symmetric and positivesemidefinite. Hence, its eigenvalues are real and nonnegative. If $\mathbf{W} \geq 0$ (where $\geq$ denotes element-wise inequality), then $\mathbf{L}_{w}$ is also symmetric, positive-semidefinite, see [8] for details.

\section{PROBLEM FORMULATION}

Consider a network of $N$ nodes communicating over a graph $\mathcal{G}$, where each node, $l$, possesses a scalar quantity, $y_{l}$. We would like to consider distributed updates on $\mathcal{G}$ of the following form: at each sensor $l$, we have

$$
x_{l}(t+1)=x_{l}(t)+h_{l}\left(\mathcal{D}_{l}\right), \quad x_{l}(0)=y_{l},
$$

where $h_{l}$ is some function such that the above algorithm converges to

$$
\lim _{t \rightarrow \infty} x_{l}(t+1)=\frac{1}{N} \sum_{j=0}^{N} x_{j}(0)=\frac{1}{N} \sum_{j=0}^{N} y_{j}, \quad \forall l,
$$

i.e., to the average of the scalar quantities, $y_{l}$, the sensors possess. The conventional average-consensus (LDAC) algorithm is linear where we choose

$$
h_{l}\left(\mathcal{D}_{l}\right)=-\mu \sum_{j \in \mathcal{K}(l)}\left(x_{l}(t)-x_{j}(t)\right), \quad \forall l .
$$

In this paper, we allow the functions, $h_{l}$, to be non-linear. In particular, we choose

$$
h_{l}\left(\mathcal{D}_{l}\right)=-\mu \sum_{j \in \mathcal{K}(l)} \sin \left(x_{l}(t)-x_{j}(t)\right), \quad \forall l .
$$

The above algorithm falls into a general class of non-linear distributed iterative algorithms that we present elsewhere. We show that the iterative algorithm (5) converges to (6) when we choose the functions $h_{l}$ to be of the form (8) under some conditions on $\mu$ and on the network connectivity. In the rest of the paper, we prove this result and establish the conditions required for convergence.

\section{NLDAC ALGORITHM}

In this section, we derive some important results relating to our algorithm. This will be helpful in proving the convergence to the average of the initial conditions. Recall from the previous section, the NLDAC is given by the following update at sensor $l$

$$
x_{l}(t+1)=x_{l}(t)-\mu \sum_{j \in \mathcal{K}(l)} \sin \left(x_{l}(t)-x_{j}(t)\right),
$$

with $x_{l}(0)=y_{l}$. We now write the above algorithm in matrix form. Define

$$
\begin{aligned}
\mathbf{x}(t) & \triangleq\left[x_{1}, \ldots, x_{N}(t)\right]^{T} \\
\mathbf{f}(\mathbf{x}(t)) & \triangleq\left[f_{1}(\mathbf{x}(t)), \ldots, f_{N}(\mathbf{x}(t))\right]^{T},
\end{aligned}
$$

where $f_{l}(\mathbf{x}(t))$ is defined as

$$
f_{l}(\mathbf{x}(t)) \triangleq x_{l}(t)-\mu \sum_{j \in \mathcal{K}(l)} \sin \left(x_{l}(t)-x_{j}(t)\right) .
$$

With the above notation, algorithm (9) can be written compactly as

$$
\mathbf{x}(t+1)=\mathbf{f}(\mathbf{x}(t))
$$

\section{A. Important results}

We now explore some properties of the function $\mathbf{f}: \mathbb{R}^{N} \rightarrow$ $\mathbb{R}^{N}$. We have the following lemma.

Lemma 1: The functions $f_{l}$ are sum preserving, i.e.,

$$
\sum_{l} f_{l}(\mathbf{x}(t))=\sum_{l} x_{l}(t)=\sum_{l} y_{l}
$$

Proof: We start with the L.H.S of (14). We have

$$
\sum_{l} f_{l}(\mathbf{x}(t))=\sum_{l}\left(x_{l}(t)-\mu \sum_{j \in \mathcal{K}(l)} \sin \left(x_{l}(t)-x_{j}(t)\right)\right) .
$$

In order to establish (14), it suffices to show that

$$
\sum_{l} \mu \sum_{j \in \mathcal{K}(l)} \sin \left(x_{l}(t)-x_{j}(t)\right)=0 .
$$

Since the communication graph is symmetric, we have

$$
j \in \mathcal{K}(l) \Rightarrow l \in \mathcal{K}(j) .
$$

Fix an $l$ and a $j \in \mathcal{K}(l)$, then there are two terms in the L.H.S of (16) that contain both $l$ and $j$. For these two terms, we have

$$
\mu \sin \left(x_{l}(t)-x_{j}(t)\right)+\mu \sin \left(x_{j}(t)-x_{l}(t)\right)=0,
$$

due to the fact that sine is an odd function. The above argument is true $\forall l$, and hence, (14) follows. Clearly, from (14), we also have

$$
\sum_{l} x_{l}(t+1)=\sum_{l} x_{l}(t) .
$$

In the following lemma, we establish the fixed point of the algorithm (13). 
Lemma 2: Let 1 denote an $N \times 1$ column-vector of 1's. For any $c \in \mathbb{R}, \mathbf{x}^{*}=c \mathbf{1}$ is a fixed point of (13).

Proof: The proof is straightforward and relies on the fact that $\sin (0)=0$.

To provide the next result, we let $\mathbf{D}(\mathbf{x})$ be a $K \times K$ diagonal matrix such that the $k$ th element on its diagonal is $\cos \left(x_{i}-x_{j}\right)$, where $i$ and $j$ are those vertices that represent the edge described by the $k$ th column of the incidence matrix, C.

Lemma 3: Let the derivative of the function $\mathbf{f}(\mathbf{x})$ with respect to $\mathbf{x}$ be denoted by $\mathbf{f}^{\prime}(\mathbf{x})$, i.e.,

$$
\mathbf{f}^{\prime}(\mathbf{x})=\frac{\partial \mathbf{f}(\mathbf{x})}{\partial \mathbf{x}}=\left\{\frac{\partial \mathbf{f}_{i}(\mathbf{x})}{\partial x_{j}}\right\}_{i, j=1, \ldots, N},
$$

then

Proof: Note that

$$
\mathbf{f}^{\prime}(\mathbf{x})=\mathbf{I}-\mu \mathbf{C D}(\mathbf{x}) \mathbf{C}^{T}
$$

$$
\frac{\partial \mathbf{f}_{i}(\mathbf{x})}{\partial x_{j}}= \begin{cases}1-\mu \sum_{j \in \mathcal{K}(i)} \cos \left(x_{i}-x_{j}\right), & i=j, \\ \mu \cos \left(x_{i}-x_{j}\right) & i \neq j,(i, j) \in \mathcal{G} \\ 0, & i \neq j,(i, j) \notin \mathcal{G} .\end{cases}
$$

With the above, we note that

$$
\left\{\frac{\mathbf{f}^{\prime}(\mathbf{x})-\mathbf{I}}{-\mu}\right\}_{i j}= \begin{cases}\sum_{j \in \mathcal{K}(i)} \cos \left(x_{i}-x_{j}\right), & i=j, \\ -\cos \left(x_{i}-x_{j}\right) & i \neq j,(i, j) \in \mathcal{G}, \\ 0, & i \neq j,(i, j) \notin \mathcal{G} .\end{cases}
$$

is a weighted Laplacian matrix with the corresponding weight for each edge, $(i, j) \in \mathcal{G}$, coming from the matrix $\mathbf{D}(x)$.

\section{B. Error Analysis}

In this subsection, we present the error propagation and provide an upper bound on the error norm. Let

$$
x_{\text {avg }}=\frac{1}{N} \sum_{l=1}^{N} x_{l}(0) \text {. }
$$

Define the error in the iterations (13) as

$$
\mathbf{e}(t+1) \triangleq \mathbf{x}(t+1)-x_{\operatorname{avg}} \mathbf{1}
$$

The following lemma provides an upper bound on the norm of $\mathbf{e}(t)$.

Lemma 4: Let $\mathbf{J}=\frac{\mathbf{1 1}^{T}}{N}$. Then, we have for some $\theta(t) \in$ $[0,1]$

$$
\|\mathbf{e}(t+1)\| \leq\left\|\mathbf{I}-\mu \mathbf{C D}(\theta(\mathbf{t}) \mathbf{e}(\mathbf{t})) \mathbf{C}^{T}-\mathbf{J}\right\|\|\mathbf{e}(t)\| .
$$

Proof: Note that

$$
\mathbf{J} x_{\operatorname{avg}} \mathbf{1}=x_{\operatorname{avg} \mathbf{1},} \mathbf{J x}(t)=x_{\operatorname{avg}} \mathbf{1},
$$

where the first equation uses $\mathbf{1}^{T} \mathbf{1}=N$, and the second equation is a consequence of Lemma 1. Also note from Lemma 2 that $\mathbf{f}\left(x_{\operatorname{avg} 1)} \mathbf{1}=x_{\operatorname{avg} 1}\right.$. From (25), (13), and (27), we have

$$
\begin{aligned}
\mathbf{e}(t+1) & =\mathbf{f}(\mathbf{x}(t))-\mathbf{f}\left(x_{\operatorname{avg}} \mathbf{1}\right)-\mathbf{J}\left(\mathbf{x}(t)-x_{\operatorname{avg} \mathbf{1})}\right. \\
& =\mathbf{g}(\mathbf{x}(t))-\mathbf{g}\left(x_{\operatorname{avg}} \mathbf{1}\right)
\end{aligned}
$$

where $\mathbf{g}: \mathbb{R}^{N} \rightarrow \mathbb{R}^{N}$ is defined as $\mathbf{g}(\mathbf{x})=\mathbf{f}(\mathbf{x})-\mathbf{J} \mathbf{x}$. The error norm is, thus, given by

$$
\begin{aligned}
\|\mathbf{e}(t+1)\| & =\left\|\mathbf{g}(\mathbf{x}(t))-\mathbf{g}\left(x_{\operatorname{avg}} \mathbf{1}\right)\right\|, \\
& =\| \mathbf{g}^{\prime}(\eta(t))(\mathbf{x}(t))-x_{\operatorname{avg} \mathbf{1}) \|,} \\
& \leq\left\|\mathbf{g}^{\prime}(\eta(t))\right\|\|\mathbf{e}(t)\|, \\
& =\left\|\mathbf{I}-\mu \mathbf{C D}(\eta(\mathbf{t})) \mathbf{C}^{T}-\mathbf{J}\right\|\|\mathbf{e}(t)\|,
\end{aligned}
$$

where the second equation follows from the mean value theorem and the fact that $\mathbf{g}$ is continuously differentiable, so that there exists some $\theta(t) \in[0,1]$ where $\eta(t)=\theta(t) \mathbf{x}(t)+$ $(1-\theta(t)) x_{\operatorname{avg}} \mathbf{1}$. The last equation follows from the definition of $g$ and Lemma 3.

Recall that the elements of $\mathbf{D}(\eta(t))$ are of the form $\cos \left(\eta_{i}(t)-\eta_{j}(t)\right)$. We have

$$
\begin{aligned}
\eta_{i}(t)-\eta_{j}(t) & =\theta(t) x_{i}(t)+(1-\theta(t)) x_{\mathrm{avg}} \\
& -\left(\theta(t) x_{j}(t)+(1-\theta(t)) x_{\mathrm{avg}}\right) \\
& =\theta(t)\left(x_{i}(t)-x_{j}(t)\right) .
\end{aligned}
$$

Hence, we can write $\mathbf{D}(\eta(t))$ as $\mathbf{D}(\theta(t) \mathbf{e}(t))$ and (26) follows.

We introduce the following notation, which we will use in presenting the main result of this paper. Let

$$
\mathbf{L}_{t}=\mathbf{C D}(\theta(t) \mathbf{e}(t)) \mathbf{C}^{T}
$$

Define $\mathcal{L}$ as the set of all possible $\mathbf{L}_{t}$ when the algorithm is initialized with the initial conditions, $x^{\prime}(0)$, i.e.,

$$
\mathcal{L}=\left\{\mathbf{L}_{t} \mid \mathbf{x}(0)=\mathbf{x}^{\prime}(0)\right\} .
$$

Recall that the weighted Laplacian, $\mathbf{L}_{t}$, is symmetric, positive-semidefinite, when the diagonal matrix, $\mathbf{D}(\theta(t) \mathbf{e}(t))$, is non-negative. Let $\mathbf{Q}_{t}=$ $\left[\mathbf{q}_{1}(t), \mathbf{q}_{2}(t), \ldots, \mathbf{q}_{N}(t)\right]$ be the matrix of $N$ linearly independent eigenvectors of $\mathbf{L}_{t}$ with the corresponding eigenvalues denoted by $\lambda_{i}\left(\mathbf{L}_{t}\right), i=1, \ldots, N$. Without loss of generality, we assume that ${ }^{1} \lambda_{1} \leq \lambda_{2} \leq \ldots \leq \lambda_{N}$, and $\mathbf{q}_{1}(t)=\mathbf{1}$ with the corresponding eigenvalue $\lambda_{1}\left(\mathbf{L}_{t}\right)=0$. Define

$$
\begin{aligned}
p_{2} & \triangleq \inf _{\mathcal{L}} \lambda_{2}\left(\mathbf{L}_{t}\right), \\
p_{N} & \triangleq \sup _{\mathcal{L}} \lambda_{N}\left(\mathbf{L}_{t}\right) .
\end{aligned}
$$

Lemma 5: The eigenvectors of the matrix $\mathbf{I}-\mu \mathbf{L}_{t}-\mathbf{J}$ are the column vectors in the matrix $\mathbf{Q}_{t}$ and the corresponding eigenvalues are 0 and $1-\mu \lambda_{i}\left(\mathbf{L}_{t}\right), i=2, \ldots, N$.

Proof: The first eigenvector of the matrix $\mathbf{I}-\mu \mathbf{L}_{t}-\mathbf{J}$ is $\mathbf{q}_{1}(t)=\mathbf{1}$ with the eigenvalue 0 . This can be shown as

$$
\left(\mathbf{I}-\mu \mathbf{L}_{t}-\mathbf{J}\right) \mathbf{q}_{1}=\mathbf{1}-\mu \mathbf{L}_{t} \mathbf{1}-\mathbf{J} \mathbf{1}=\mathbf{0} .
$$

That the rest of the eigenvectors, $\mathbf{q}_{2}(t), \ldots, \mathbf{q}_{N}(t)$, of $\mathbf{L}_{t}$, are also the eigenvectors of $\mathbf{I}-\mu \mathbf{L}_{t}-\mathbf{J}$ can be established by the fact that $\mathbf{J}$ is rank 1 with eigenvector $\mathbf{1}$ and the identity matrix can have any set of linearly independent vectors as its eigenvectors.

\footnotetext{
${ }^{1}$ Note that $\mathbf{L}_{t}$ is symmetric, positive-semidefinite so its eigenvalues are positive reals.
} 
The next lemma establishes that $x_{l}(t) \in[-\pi / 4+\epsilon, \pi / 4-$ $\epsilon], \forall l, t$, when the initial condition, $\mathbf{x}(0)$, lies in the same range.

Lemma 6: Let the vector of network initial conditions, $\mathbf{x}(0)$, be such that

$$
x_{l}(0) \in[-\pi / 4+\epsilon, \pi / 4-\epsilon], \forall l,
$$

where $\epsilon>0$ is a sufficiently small real number. Then for $\mu$ in the range $0<\mu \leq \frac{\pi}{2 d_{\max }}$, we have

$$
x_{l}(t) \in[-\pi / 4+\epsilon, \pi / 4-\epsilon], \forall l, t .
$$

Proof: We use the following bound to prove this lemma.

$$
\frac{2}{\pi} x \leq \sin (x) \leq x, \quad 0 \leq x \leq \frac{\pi}{2} .
$$

For any arbitrary node, $l$, partition its neighbors, $\mathcal{K}(l)$, into $\mathcal{K}_{L}(l, t)$ and $\mathcal{K}_{G}(l, t)$, where $\mathcal{K}_{L}(l, t)=\left\{j \in \mathcal{K}(l) \mid x_{l}(t)>\right.$ $\left.x_{j}(t)\right\}$, and $\mathcal{K}_{G}(l, t)=\left\{j \in \mathcal{K}(l) \mid x_{l}(t)<x_{j}(t)\right\}$. We can write the NLDAC iterations (9) as

$$
\begin{aligned}
x_{l}(t+1) & =x_{l}(t)-\mu \sum_{j \in \mathcal{K}_{L}(l, t)} \sin \left(x_{l}(t)-x_{j}(t)\right) \\
& +\mu \sum_{j \in \mathcal{K}_{G}(l, t)} \sin \left(x_{j}(t)-x_{l}(t)\right), \\
& \leq x_{l}(t)-\mu \sum_{j \in \mathcal{K}_{L}(l, t)} \frac{2}{\pi}\left(x_{l}(t)-x_{j}(t)\right) \\
& +\mu \sum_{j \in \mathcal{K}_{G}(l, t)}\left(x_{j}(t)-x_{l}(t)\right), \\
& =\left(1-\frac{2 \mu}{\pi}\left|\mathcal{K}_{L}(l, t)\right|-\mu\left|\mathcal{K}_{G}(l, t)\right|\right) x_{l}(t) \\
& +\frac{2 \mu}{\pi} \sum_{j \in \mathcal{K}_{L}(l, t)} x_{j}(t)+\mu \sum_{j \in \mathcal{K}_{G}(l, t)} x_{j}(t),(39)
\end{aligned}
$$

where the inequality follows from (37) and the bound in (38). Similarly, we can show

$$
\begin{aligned}
x_{l}(t+1) & \geq x_{l}(t)-\mu \sum_{j \in \mathcal{K}_{L}(l, t)}\left(x_{l}(t)-x_{j}(t)\right) \\
& +\mu \sum_{j \in \mathcal{K}_{G}(l, t)} \frac{2}{\pi}\left(x_{j}(t)-x_{l}(t)\right), \\
& =\left(1-\mu\left|\mathcal{K}_{L}(l, t)\right|-\frac{2 \mu}{\pi}\left|\mathcal{K}_{G}(l, t)\right|\right) x_{l}(t) \\
& +\mu \sum_{j \in \mathcal{K}_{L}(l, t)} x_{j}(t)+\frac{2 \mu}{\pi} \sum_{j \in \mathcal{K}_{G}(l, t)} x_{j}(t) .(40)
\end{aligned}
$$

Combining (39) and (40), $x_{l}(t+1)$ remains bounded above and below by a convex combination of $x_{j}(t), j \in \mathcal{D}_{l}$, when

$$
\begin{aligned}
& 0 \leq \frac{2 \mu}{\pi}\left|\mathcal{K}_{L}(l, t)\right|+\mu\left|\mathcal{K}_{G}(l, t)\right| \leq 1 \\
& 0 \leq \mu\left|\mathcal{K}_{L}(l, t)\right|+\frac{2 \mu}{\pi}\left|\mathcal{K}_{G}(l, t)\right| \leq 1
\end{aligned}
$$

The L.H.S is trivially satisfied in both of the above equations. To derive $\mu$ such that the R.H.S is satisfied for both of the above equations, we use

$$
\begin{aligned}
\mu & \leq \frac{1}{\frac{2}{\pi}\left(\mathcal{K}_{L}(l, t)|+| \mathcal{K}_{G}(l, t) \mid\right)} \\
& \leq \frac{\pi}{2 d_{\max }} .
\end{aligned}
$$

With $\mu$ satisfying the above equation, $x_{l}(t+1)$ is bounded above and below by a convex combinations of $x_{j}(t), j \in$ $\mathcal{D}(l)$. So, if $x_{j}(t) \in[-\pi / 4+\epsilon, \pi / 4-\epsilon]$, for $j \in \mathcal{D}(l)$, so does its convex combinations and thus $x_{l}(t+1) \in[-\pi / 4+$ $\epsilon, \pi / 4-\epsilon]$ and the lemma follows.

Using the above lemma, we now have the following result.

Lemma 7: Let (37) hold. Let the network communication graph be connected, i.e., $\lambda_{2}(\mathbf{L})>0$, then $p_{2}>0$, for $0<$ $\mu \leq \pi / 2 d_{\max }$.

Proof: Consider $\mathbf{L}_{w}$ as defined in (4) with $\mathbf{W}>0$ (element-wise inequality, also recall $\mathbf{W}$ is a $K \times K$ diagonal matrix with $w_{k}^{k=1, \ldots, K}=w_{i j}$ on its diagonal denoting the weight of the $k$ th edge, $(i, j) \in \mathcal{G})$. We have

$$
\mathbf{z}^{T} \mathbf{L}_{w} \mathbf{z}=\sum_{(i, j) \in \mathcal{G}} w_{i j}\left(z_{i}-z_{j}\right)^{2}
$$

for any $\mathbf{z} \in \mathbb{R}^{N}$. Since $w_{i j}>0, \forall(i, j) \in \mathcal{G}$, the quadratic form (45) is 0 if and only if $\mathbf{z}=c \mathbf{1}$, for any $c \in \mathbb{R}$. So $\mathbf{L}_{w}$ has only one eigenvalue of 0 with eigenvector $\mathbf{1}$. Hence, $\lambda_{2}\left(\mathbf{L}_{\mathbf{w}}\right)>0$. Also, note that $\lambda_{2}\left(\mathbf{L}_{\mathbf{w}}\right)$ is a continuous function of $w_{i j}$ 's [9]. So the infimum of $\lambda_{2}\left(\mathbf{L}_{\mathbf{w}}\right)$ is attainable if the elements of $\mathbf{W}$ lie in a compact set. To this end, define

$\mathcal{C}=\left\{\mathbf{W} \in \mathbb{R}^{K \times K} \mid w_{i i} \in[\cos (\pi / 2-2 \epsilon), 1], w_{i j}=0(i \neq j)\right\}$,

and note that $\mathbf{D}(\theta(t) \mathbf{e}(t)) \in \mathcal{C}$ from Lemma 6. Since $\mathbf{L}_{t}=$ $\mathbf{C D}(\theta(t) \mathbf{e}(t)) \mathbf{C}^{T}$ and $\mathbf{D}(\theta(t) \mathbf{e}(t)) \in \mathcal{C}$, we note that

$$
p_{2}=\inf _{\mathcal{L}} \lambda_{2}\left(\mathbf{L}_{t}\right) \geq \inf _{\mathbf{W} \in \mathcal{C}} \lambda_{2}\left(\mathbf{L}_{w}\right) .
$$

We now use a contradiction argument to show $p_{2}>0$. Assume on the contrary that $p_{2}=0$. Then $\inf _{\mathbf{W} \in \mathcal{C}} \lambda_{2}\left(\mathbf{L}_{w}\right)=$ 0 and there exists some $\mathbf{W} \in \mathcal{C}$ such that $\lambda_{2}\left(\mathbf{L}_{w}\right)=0$. But, for all $\mathbf{W} \in \mathcal{C}$, we have $\lambda_{2}\left(\mathbf{L}_{w}\right)>0$, which is a contradiction. Hence, $p_{2}>0$.

We now present the convergence of NLDAC in the following theorem.

Theorem 1: Let the vector of network initial conditions be denoted by $\mathbf{x}(0)$ such that (37) holds. Let the network communication graph, $\mathcal{G}$, be connected, i.e., $\lambda_{2}(\mathbf{L})>0$. If $\mu$ is such that

$$
0<\mu<\frac{2}{p_{N}}
$$

then

$$
\lim _{t \rightarrow \infty}\|\mathbf{e}(t)\|=\mathbf{0}
$$

Proof: From (34) and (48), we have for $i=2, \ldots, N$

$$
1-\mu \lambda_{i}\left(\mathbf{L}_{t}\right) \geq 1-\mu p_{N}>1-\frac{2}{p_{N}} p_{N}=-1 \text {. }
$$

From (33), we have for $i=2, \ldots, N$

$$
1-\mu \lambda_{i}\left(\mathbf{L}_{t}\right) \leq 1-\mu p_{2}<1,
$$




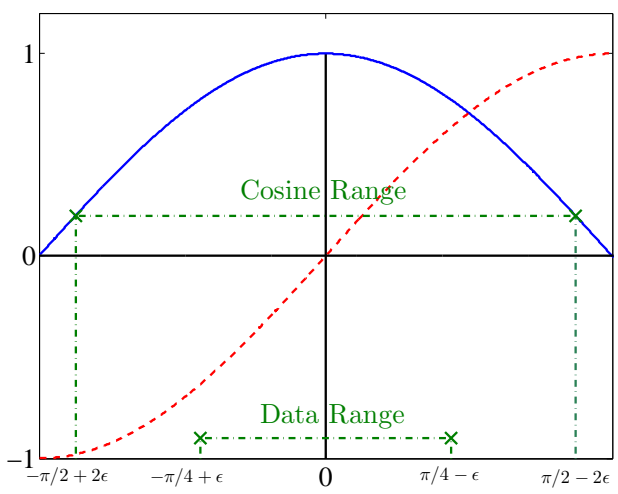

Fig. 1. Figure corresponding to Remark (i).

from (48) the fact that $p_{2}>0$ from Lemma 7. Combining (50) and (51), we have

$$
-1<1-\mu \lambda_{i}\left(\mathbf{L}_{t}\right)<1, \quad i=2, \ldots, N,
$$

With the above, we have $\left|1-\mu \lambda_{i}\left(\mathbf{L}_{t}\right)\right|<c<1, i=$ $2, \ldots, N$ and for some $c \in[0,1)$. Thus, the error norm is given by

$$
\|\mathbf{e}(t+1)\| \leq \max _{2 \leq i \leq N}\left|1-\mu \lambda_{i}\left(\mathbf{L}_{t}\right)\right|\|\mathbf{e}(t)\|<c\|\mathbf{e}(t)\|,
$$

and (49) follows.

We, further, have $p_{N} \leq 2 d_{\max }$ [8], where $d_{\max }$ is the maximum degree of the graph generated by the Laplacian, $\mathbf{L}=\mathbf{C C}^{T}$. We now have convergence for

$$
0<\mu<\frac{1}{d_{\max }} \leq \frac{2}{p_{N}} .
$$

Remarks: We now make some relevant remarks.

(i) We explain our assumption in (37) with the help of Fig. 1. When the data, $x_{l}(0), \forall l$, lies in the interval $[-\pi / 4+$ $\epsilon, \pi / 4-\epsilon]$, then $x_{l}(t)-x_{j}(t)$ for all $t$ and $l \neq j$ must lie in the interval $[-\pi / 2+2 \epsilon, \pi / 2-2 \epsilon]$. This is true for all $t$, since the choice of $\mu$ in (48) guarantees a contraction from Theorem 1. Hence, $\cos \left(x_{l}(t)-x_{j}(t)\right), \forall t$, must lie in the interval $[\cos (\pi / 2-2 \epsilon), 1]$, which is strictly greater than 0 for $\epsilon>0$, as shown in Fig. 1. With $\cos \left(x_{l}(t)-x_{j}(t)\right) \in$ $[\cos (\pi / 2-2 \epsilon), 1], \forall t$, we note that $\mathbf{L}_{t}$ does not lose the sparsity (zero-one) pattern of $\mathbf{L}$ and hence, $\lambda_{2}\left(\mathbf{L}_{t}\right)>0, \forall t$.

Clearly, if (37) does not hold but the initial data has a known bound, we can introduce a frequency parameter, $\zeta$, in the sine function such that $\cos \left(\zeta\left(x_{l}(t)-x_{j}(t)\right)\right) \in$ $[\cos (\pi / 2-2 \epsilon), 1]$. Hence, the assumption in (48) does not lose generality.

(ii) Note that $p_{N}$ may not be known or easily computable a priori.In that case, one may work with $0<\mu<1 / d_{\max }$ as established in (54), which is readily determined.

(iii) Choosing $\mu$ away from the bound in (54), results into a divergence of the algorithm, as we will elaborate in the simulations. This is a manifestation of the bifurcation phenomena as arise in the non-linear theory.

\section{Simulations}

We consider a network of $N=100$ nodes, shown in Fig. 2(a). We implement the conventional linear distributed average-consensus algorithm with optimal constant weights, i.e., we choose

$$
\mu_{\mathrm{LIN}}^{\mathrm{oPT}}=\frac{2}{\lambda_{2}(\mathbf{L})+\lambda_{N}(\mathbf{L})},
$$

in (7). The error norm in this case is shown in Fig. 2(b) as a red dotted curve. To show the performance of the NLDAC algorithm (9), we choose the following values of $\mu$

$$
\mu=\left\{\frac{0.99}{d_{\max }}, \frac{2}{d_{\max }}, \frac{1}{2 d_{\max }}, \frac{1}{3 d_{\max }}\right\}
$$

and show the error norm in Fig. 2(b) and Fig. 2(c).

\section{CONCLUSIONS}

In this paper, we present a non-linear distributed averageconsensus (NLDAC) algorithm that uses the sine of the state differences among the nodes instead of the conventional linear update. The convergence rate of the NLDAC algorithm now depends on the cosine of the state differences (as cosine is the derivative of the sine) and, thus, depends on the actual state values. Due to this dependence, the convergence rate has an additional degree of freedom as the convergence rate in LDAC only depends on the network connectivity. We provide simulations to assert the theoretical findings.

\section{REFERENCES}

[1] L. Xiao and S. Boyd, "Fast linear iterations for distributed averaging," Systems and Controls Letters, vol. 53, no. 1, pp. 65-78, Apr. 2004.

[2] R. Olfati-Saber, J. A. Fax, and R. M. Murray, "Consensus and cooperation in networked multi-agent systems," Proceedings of the IEEE, vol. 95, no. 1, pp. 215-233, Jan. 2007.

[3] Y. Kuramoto, "Cooperative dynamics of oscillator community," Progress of Theoretical Physics Suppl., vol. 79, pp. 223-240, 1984.

[4] C. W. Wu and L. O. Chua, "Synchronization in an array of linearly coupled dynamical systems," IEEE Transactions on Circuits Systems I, vol. 42, pp. 430-447, Aug. 1995.

[5] A. Mauroy and R. Sepulchre, "Clustering behaviors in networks of integrate-and-fire oscillators," Chaos: Special issue on Synchronization in Complex Networks, vol. 18, no. 3, pp. 037122-037122-8, 2008.

[6] N. Varanese, O. Simeone, U. Spagnolini, and Y. Bar-Ness, "Distributed frequency-locked loops for wireless networks," in 10th IEEE International Symposium on Spread Spectrum Techniques and Applications, Bologna, Italy, Aug. 2008, pp. 400-404.

[7] A. Bergen and D. Hill, "A structure preserving model for power system stability analysis," IEEE Trans. on Power Apparatus and Systems, vol. PAS-100, no. 1, pp. 25-35, Jan. 1981.

[8] F. R. K. Chung, Spectral Graph Theory, Providence, RI : American Mathematical Society, 1997.

[9] F. R. Gantmacher, Matrix Theory (Volume I), Chelsea Publishing Co., USA, 1959. 


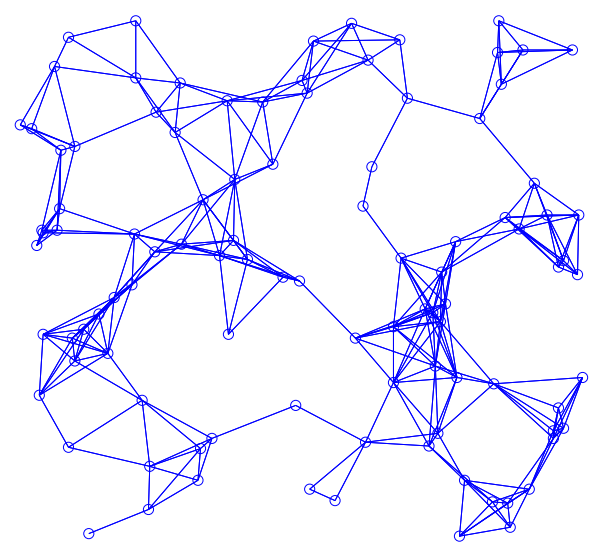

(a)

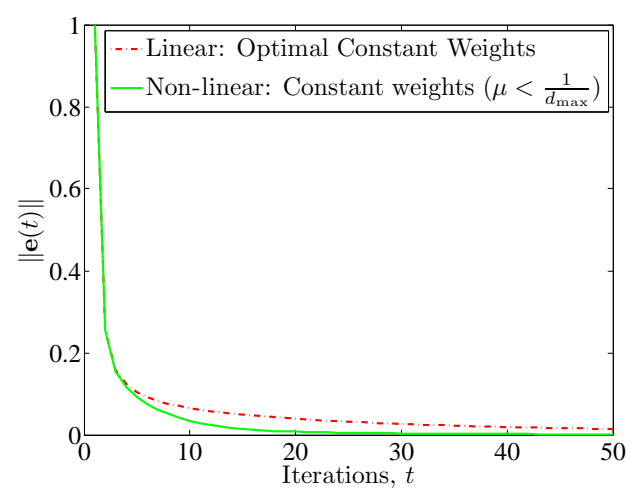

(b)

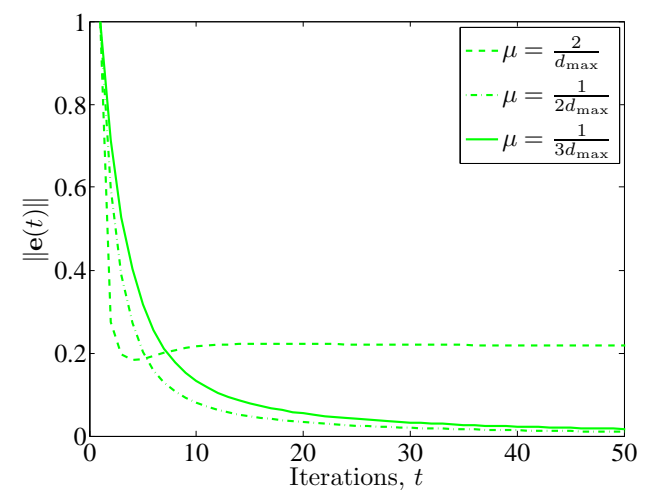

(c)

Fig. 2. (a) An $N=100$ node network. (b) Comparison of the NLDAC with LDAC using constant optimal edge weights. (c) The error norm of NLDAC for different values of $\mu$. 